\title{
Metalosato de zinc en respuesta agronómica y composición química del pasto mombaza en la amazonía ecuatoriana
}

\section{Metalosato zinc in agronomic response and chemical composition of grass mombaza in ecuadorian amazon}

${ }^{\circ}$ Pedro Eduardo Nivela Morante ${ }^{1}$, Juan Humberto Avellaneda Cevallos², Manuel de Jesús Jumbo Romero ${ }^{1}$, Laura Morante Carriel $^{3}$, Yosbel Lazo Roger ${ }^{1}$, Jefferson Gustavo Aragundi Velarde ${ }^{4}$

${ }^{1}$ Universidad Laica Eloy Alfaro de Manabi. Av. Circunvalación - Vía a San Mateo. Manta, Manabí, Ecuador. •eduquevedo2011@hotmail.com; manueldejesusjumboromero@hotmail.com; ylazo@gmail.com

${ }^{2}$ Universidad Técnológica Equinoccial. Facultad de Ciencias de la Ingeniería e Industrias. Maestría en Zootecnia mención Nutrición Animal. Ssede Santo Domingo. Santo Domingo de los Tsachilas, km 4 1/2 vía Chone, Casilla Postal 17-01-2764. Santo Domingo,Ecuador. javellaneda@ute.edu.ec

${ }^{3}$ Universidad Técnica Estatal de Quevedo. Facultad de Ciencias Ambientales. Carrera de Ingeniería Ambiental. Campus Ing. Manuel Haz Álvarez, km 1.5 vía a Santo Domingo de los Tsáchilas. EC.120501.Quevedo, Ecuador. lamor424@gmail.com

${ }^{4}$ Universidad Técnica Estatal de Quevedo. Facultad de Ciencias Agrarias. Carrera de Ingeniería Agronómica. Campus Ing. Manuel Haz Álvarez, km 1.5 vía a Santo Domingo de los Tsáchilas. EC.120501.Quevedo, Ecuador. jaragundi@uteq.edu.ec

Rec.: 22.05.2017. Acept.: 08.09.2017. Publicado el 1 de diciembre de 2017

\section{Resumen}

$\mathrm{E}$ n este estudio se pretendió mejorar el comportamiento agronómico del pasto mombaza con fertilización complementaria de metalosato de zinc, con la finalidad de elevar los parámetros productivos y nutritivos del pasto. La investigación se realizó en la provincia de Orellana, cantón Joya de los Sachas. Se aplicó un diseño completamente al azar (DCA) con arreglo factorial 3 (metalosato de zinc 0, 1, y 2 litros ha-1) x 3 (edad de cosecha 28, 35 y 42 días). Las variables en estudio fueron peso de hoja $(\mathrm{g})$, peso de tallo $(\mathrm{g})$, número de hojas, longitud de hojas $(\mathrm{cm})$, relación hoja tallo en peso (g), Biomasa kg.ha- ${ }^{-1}$, contenido de: materia seca $(\%)$, proteína $(\%)$, materia inorgánica (\%) y materia orgánica (\%). A los 42 días de corte se alcanzaron los mayores resultados en peso de hoja, peso de tallo, número de hojas, longitud de hojas, biomasa y materia seca, no obstante, a los 28 días de corte se obtuvo mayor efecto en las variables relación hoja/tallo, proteína y materia orgánica. El efecto interacción se destacó $2 \mathrm{~L} \mathrm{ha}^{-1} \mathrm{x} 42$ días de corte en todas las variables agronómicas, también se evidenció que la interacción $2 \mathrm{~L} \mathrm{ha}^{-1}$ x 28 días de corte, lograron mejor efecto en contenido de materia seca con $30.12 \%$, además en el contenido de proteínas se destacó la interacción $1 \mathrm{~L} \mathrm{ha}^{-1}$ x 28 días con $9.99 \%$ y en contenido de materia orgánica resalta $0 \mathrm{~L} \mathrm{ha}^{-1}$ x 28 días con $86.49 \%$.

Palabras clave: quelato, biomasa, pastizales.
$\mathrm{T}$ his study aimed to improve the agronomic behavior of mombaza grass with supplemental fertilization of zinc metalosate, in order to raise the productive and nutritive parameters of the pasture. The investigation was carried out in the province of Orellana, canton Joya de los Sachas. A completely randomized design (DCA) with factorial arrangement 3 (zinc metalosate 0,1 , and 2 liters ha $^{-1}$ ) x 3 (harvest age 28, 35 and 42 days) was applied. The variables studied were leaf weight $(\mathrm{g})$, stem weight $(\mathrm{g})$, number of leaves, leaf length $(\mathrm{cm})$, stem leaf weight ratio $(\mathrm{g})$, Biomass $\mathrm{kg} \cdot \mathrm{ha}^{-1}$, content of dry matter $(\%)$, protein $(\%)$, inorganic matter $(\%)$ and organic matter $(\%)$. At the 42 days of harvest, the highest results were obtained in leaf weight, stem weight, leaf number, leaf length, biomass and dry matter, but at 28 days of cut, stem, protein and organic matter. In effect interaction was highlighted $2 \mathrm{~L} \mathrm{ha}^{-1} \mathrm{x} 42$ days cut in all agronomic variables, it was also evidenced that the interaction $2 \mathrm{~L} \mathrm{ha}^{-1}$ x 28 days of cut achieved better effect on dry matter content with $30.12 \%$, in addition to the protein content, the interaction between $1 \mathrm{~L} \mathrm{ha}^{-1}$ x 28 days with $9.99 \%$ was highlighted and organic matter content stood out $0 \mathrm{~L} \mathrm{ha}^{-1} \mathrm{x}$ 28 days with $86.49 \%$.

Key words: chelate, biomass, pasture. 


\section{Introducción}

$\mathrm{L}$ a producción ganadera en los pastizales nativos e introducidos, dependen de la calidad y cantidad de forraje disponible. La mayoría de zonas áridas y semiáridas tienen periodos definidos de sequía y épocas invernales, durante los cuales la calidad y cantidad de forrajes disponibles disminuyen, especialmente la proteína cruda y la digestibilidad. Como consecuencia, se reduce el consumo de forraje, reflejándose en pérdidas de peso que disminuyen la producción (Villalobos et al., 2000; Verdecia et al., 2009). En el mundo actual, los ganaderos deben producir más eficientemente para ser competitivos en el mercado (La O et al., 2012). En el manejo de forrajes tropicales existen muchos factores que se deben tomar en cuenta para lograr una alta eficiencia en la utilización del pasto producido y la máxima producción animal por unidad de superficie (Cerdas, 2011).

El pasto Mombaza es un cultivar de la especie Panicum máximum Jacq., que por su alta capacidad de producción de biomasa, está siendo introducido como una opción para mejorar la productividad de las praderas tropicales; sin embargo, el manejo tradicional aplicado y la falta de recomendaciones particulares para esta especie, no ha permitido alcanzar el impacto esperado, y muchas de estas praderas muestran signos de degradación (Ramírez et al., 2010). Las gramíneas tropicales presentan fluctuaciones en su valor nutritivo a través del año, disminuyendo su calidad especialmente en la época seca, produciendo una deficiente respuesta animal y como consecuencia la presencia de sistemas productivos y reproductivos deficientes (Garmendia, 1998). Por ello, Verdecia et al. (2012) indica que es de gran importancia profundizar en el efecto de la edad de rebrote y los factores climáticos en el valor nutritivo de Panicum maximum, según los distintos períodos de año.

El Guinea mombaza muestra características superiores con respecto a otras que se obtuvieron y liberaron también en Brasil. Por tanto, se considera una de las especies forrajeras tropicales más productivas. Los primeros ensayos se realizaron en Paraná, donde superó a otros cultivares, demostrando alto potencial productivo para la producción de forrajes como para el pastoreo intensivo. En estas condiciones llegaron a obtenerse producciones de $33 \mathrm{t}$ de ms/ha/año (Müller et al., 2002).

Según Nowack (2002), en la actualidad los quelatos atraen poderosamente la atención a investigar por ser una excelente alternativa para adicionar metales de manera edáfica y foliar a las plantas, teniendo presente que pueden incrementar la solubilización de los metales: hierro (Fe), zinc (Zn), manganeso (Mn); transportarlo hacia la raíz y/o hoja de la planta; y una vez allí, ceder dichos metales (Fe, Zn, Mn), y la parte orgánica del quelato debe volver a solubilizar más metal.

Al utilizar este tipo de nutrición complementaria en el pasto mombaza se compensará los requerimientos de zinc involucrado en tantas enzimas ya que el zinc es crítico para el metabolismo de muchos nutrientes incluyendo proteínas, ácidos nucleicos y carbohidratos, por tanto, se considera un elemento mineral esencial para la vida. Además, los procesos enzimáticos en los que interviene el $\mathrm{Zn}$, tiene su acción principal en los tejidos de alta velocidad de formación de células, de allí que su deficiencia perjudique el crecimiento de los terneros, disminuya la producción de espermatozoides en los carneros y toros y favorezca las enfermedades de la piel (González, 2016).

El presente trabajo investigativo pretendió mejorar el comportamiento agronómico y composición química del pasto mombaza en la amazonia ecuatoriana.

\section{Materiales y métodos}

T a presente investigación se llevó en dos sitios, el primero correspondió a la fase agronómica que se realizó en la provincia de Orellana, cantón Joya de los Sachas, parroquia Lago San Pedro en la finca del Sr. Manuel Jaya Garzón y el segundo fue determinar la composición química, realizada en el laboratorio de Rumiología y Metabolismo Nutricional, ubicada en la finca experimental "La María" de la Universidad Técnica Estatal de Quevedo, Facultad de Ciencias Pecuarias, ubicada en el km. 7 vía Quevedo - El Empalme, provincia de Los Ríos. La ubicación geográfica es de $01^{\circ} 0.6^{\circ}$ de latitud sur y $79^{\circ} 29^{\circ}$ de latitud oeste, con una altura de $73 \mathrm{msnm}$.

En el comportamiento agronómico y composición química se utilizó un diseño completo al azar (DCA) con un arreglo factorial $(3 \times 3)$. Se empleó un total de 9 tratamientos y se utilizó tres repeticiones. El factor y variables de estudio en comportamiento agronómico y composición química son los Niveles de metalosato de zinc (0, 1, y 2 litros) y edad de cosecha (28, 35 y 42 días). Las variables en estudio: peso de hoja (g), peso de tallo (g), número de hojas, longitud de hojas $(\mathrm{cm})$, relación hoja tallo en peso $(\mathrm{g})$, rendimiento $\mathrm{kg} \cdot \mathrm{ha}^{-1}$ (biomasa), contenido de materia seca (\%), contenido de proteína $(\%)$, contenido de materia inorgánica (\%) y contenido de materia orgánica $(\%)$.

Se realizó la prueba de significación de Tukey al 5\%, para cada factor en estudio y para tratamientos. Para el procesamiento de la información se utilizó el software estadístico INFOSTAT versión 2008 (Di Rienzo et al., 2008).

\section{Resultados y discusión}

\section{Comportamiento agronómico}

Efecto metalosato de zinc. La respuesta agronómica por el uso del metalosato de zinc generó un efecto altamente significativo $(p<0.01)$ en las variables agroproductivas evaluadas (Cuadro 1). Se observó que el nivel 2 L.ha $^{-1}$ sobresalió de los demás 
Cuadro 1. Efecto metalosato de zinc sobre el comportamiento agronómico del pasto mombaza (Panicum máximum Jacq)

\begin{tabular}{|c|c|c|c|c|c|}
\hline \multirow{2}{*}{ Variable } & \multicolumn{3}{|c|}{ Niveles de metalosato de zinc $\left(\mathrm{L} \mathrm{ha}^{-1}\right)$} & \multirow{2}{*}{$\mathbf{P}<$} & \multirow{2}{*}{ CV $(\%)$} \\
\hline & $\mathbf{0}$ & 1 & 2 & & \\
\hline Peso de hojas (g) & $3.88 \mathrm{~b}$ & $4.08 \mathrm{~b}$ & $6.04 \mathrm{a}$ & 0.000 & 3.80 \\
\hline Peso de tallos (g) & $3.24 \mathrm{c}$ & $4.19 \mathrm{~b}$ & $5.52 \mathrm{a}$ & 0.000 & 3.81 \\
\hline Relación hoja/tallo (g) & $1.21 \mathrm{a}$ & $0.98 \mathrm{c}$ & $1.09 \mathrm{~b}$ & 0.000 & 6.65 \\
\hline Número de hojas & $5.40 \mathrm{c}$ & $5.61 \mathrm{~b}$ & $6.29 \mathrm{a}$ & 0.000 & 2.65 \\
\hline Longitud de hojas $(\mathrm{cm})$ & $39.44 \mathrm{c}$ & $47.16 \mathrm{~b}$ & $57.98 \mathrm{a}$ & 0.000 & 2.40 \\
\hline Biomasa MS (kg ha-1) & $2433.84 \mathrm{c}$ & $2836.95 \mathrm{~b}$ & $3906.97 \mathrm{a}$ & 0.000 & 2.85 \\
\hline
\end{tabular}

Medias con una letra común, no son significativamente diferentes (Tukey, p>0.05)

tratamientos. No obstante, Chaves y Berrantes (1999) utilizaron metalosato de zinc a $0.50 \mathrm{~L} \mathrm{ha}^{-1}$ con adición de $\mathrm{Zn}$ granular al suelo y obtuvieron respuestas más efectiva y positiva que la aplicación vía foliar.

Efecto edad de cosecha. El efecto edades de cosecha en la respuesta agronómica (Cuadro 2) se observó diferencias $(\mathrm{p}<0.05)$ en la relación hoja/tallo a los 28 días de cosecha, mientras, las demás variables dependientes se obtuvieron diferencias $(\mathrm{p}<0.01)$ a los 42 días de cosecha, rendimientos superados por Paulino (2004), quien manifestó que el pasto mombaza logró rendimientos de 9.04 y $5.49 \mathrm{t}$ de $\mathrm{MS} \mathrm{ha}^{-1}$ en los períodos lluvioso y poco lluvioso, respectivamente, a esta misma edad de rebrote, coincidiendo con lo expresado por Vargas et al. (2014) quienes manifestaron que el pasto Panicum maximum vc mombaza es promisorio para las condiciones edafoclimáticas de la Amazonía ecuatoriana, con una elevada capacidad de crecimiento.

Efecto niveles de metalosato de zinc $\mathrm{x}$ edades de cosecha. El efecto interacción entre los niveles de metalosato de zinc $x$ edades de cosecha, tuvo una tendencia creciente en peso de hojas, peso de tallos, longitud de hojas y biomasa, lo que indica que existe dependencia de la variable respuesta con respecto al factor de estudio. Mientras, la relacion hoja/tallo y número de hojas tuvieron un comportamiento similar entre los dos factores de estudio, indicando independencia entre la variable respuesta y factor de estudio. De acuerdo a Garcia et al. (2008) el pasto mombaza se comporta de forma similar a las especies de gramíneas que se emplean en producción animal, pues, mantiene una adecuada proporción de hojas en su curva, más en periodo lluvioso, incrementándose hasta los 168 días de rebrote.

\section{Composición química}

Efecto metalosato de zinc. Éste presentó diferencias estadísticas ( $\mathrm{p}<0.05$ ) en materia seca (Cuadro 3), destacándose el nivel $2 \mathrm{~L} \mathrm{ha}^{-1}$, según García et al. (2008) se ha determinado que los rumiantes requieren de un mínimo de $7 \%$ de proteína cruda en la dieta, para que el consumo y la digestibilidad de la materia seca sean óptimos, siendo estos parámetros obtenidos

Cuadro 2. Efecto edad de cosecha sobre el comportamiento agronómico del pasto mombaza (Panicum máximum Jacq)

\begin{tabular}{lrrrrr}
\hline \multirow{2}{*}{ Variable } & \multicolumn{3}{c}{ Edades de cosecha (días) } & \multirow{2}{*}{$\mathbf{P}<$} & \multirow{2}{*}{ CV (\%) } \\
\cline { 2 - 5 } & $\mathbf{2 8}$ & $\mathbf{3 5}$ & $\mathbf{4 2}$ & & \\
\hline Peso de hojas (g) & $4.19 \mathrm{c}$ & $4.67 \mathrm{~b}$ & $5.14 \mathrm{a}$ & 0.000 & 3.80 \\
Peso de tallos (g) & $3.76 \mathrm{c}$ & $4.44 \mathrm{~b}$ & $4.76 \mathrm{a}$ & 0.000 & 3.81 \\
Relación hoja/tallo (g) & $1.11 \mathrm{a}$ & $1.05 \mathrm{~b}$ & $1.08 \mathrm{ab}$ & 0.036 & 6.65 \\
Número de hojas & $5.23 \mathrm{c}$ & $5.70 \mathrm{~b}$ & $6.37 \mathrm{a}$ & 0.000 & 2.65 \\
Longitud de hojas (cm) & $41.86 \mathrm{c}$ & $47.33 \mathrm{~b}$ & $55.39 \mathrm{a}$ & 0.000 & 2.40 \\
Biomasa MS (kg ha $\left.{ }^{-1}\right)$ & $2663.20 \mathrm{c}$ & $3144.81 \mathrm{~b}$ & $3369.76 \mathrm{a}$ & 0.000 & 2.85 \\
\hline
\end{tabular}

Medias con una letra común, no son significativamente diferentes (Tukey, p>0.05) 

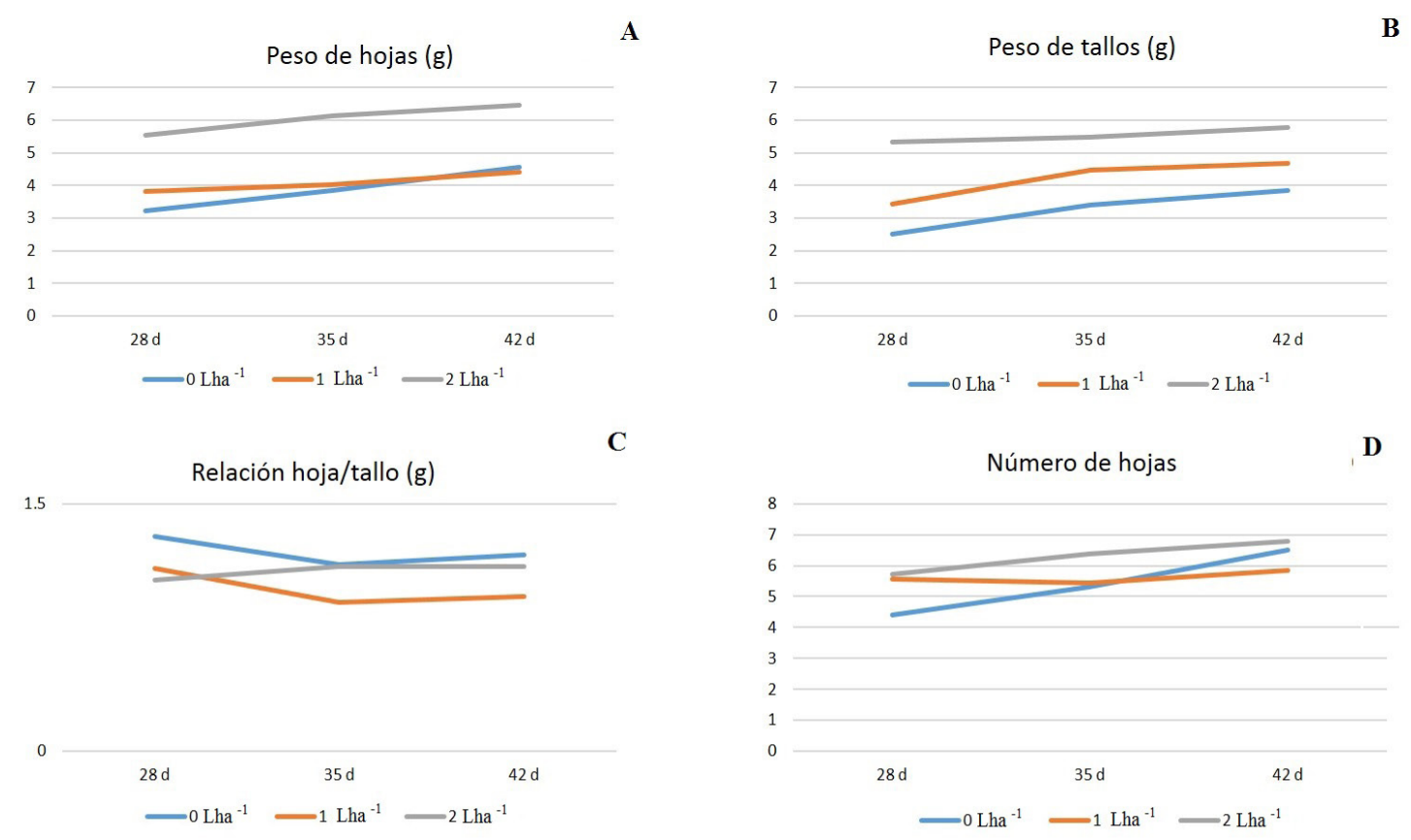

$\mathbf{E}$
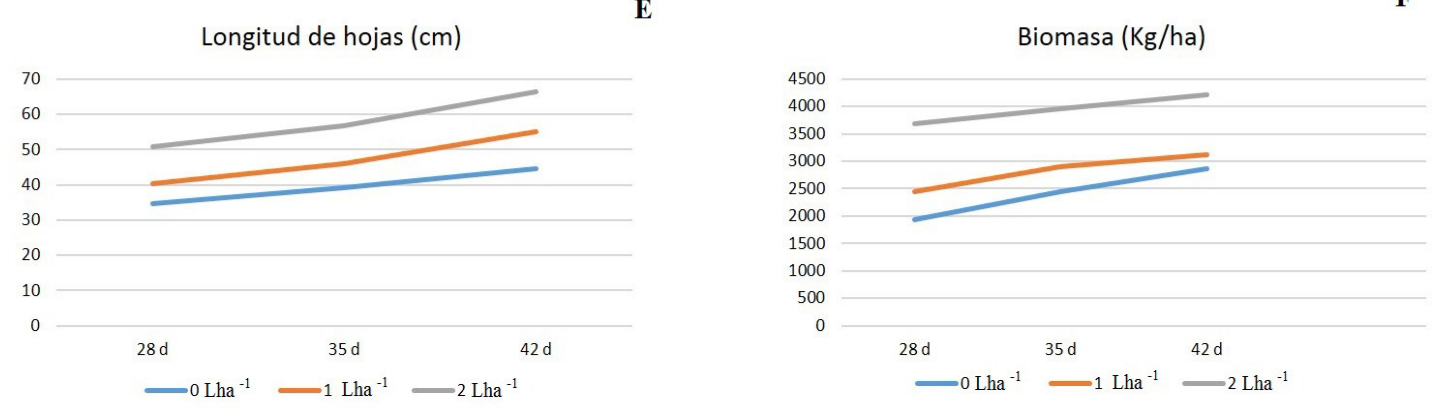

Figura 1. Comportamiento agronómico del pasto mombaza (Panicum máximum Jacq). A) efecto interacción del peso de hojas, B) peso de tallos, C) relación hojas/tallos, D) número de hojas, E) longitud de hojas y F) biomasa MS ( $\left.\mathrm{kg} \mathrm{ha}^{-1}\right)$.

Cuadro 3. Efecto del metalosato de zinc sobre la composición química del pasto mombaza (Panicum máximum Jacq)

\begin{tabular}{lrrrrr}
\hline \multirow{2}{*}{ Variable } & \multicolumn{2}{c}{ Niveles de metalosato de zinc $\mathbf{L ~ h a}^{-\mathbf{1}}$} & \multirow{2}{*}{$\mathbf{P}<$} & CV (\%) \\
\cline { 2 - 5 } & \multicolumn{1}{c}{$\mathbf{0}$} & $\mathbf{1}$ & $\mathbf{2}$ & & \\
\hline Proteína (\%) & $8.73 \mathrm{a}$ & $8.89 \mathrm{a}$ & $8.63 \mathrm{a}$ & 0.612 & 6.16 \\
MS (\%) & $29.56 \mathrm{~b}$ & $29.90 \mathrm{ab}$ & $30.03 \mathrm{a}$ & 0.017 & 0.51 \\
Ceniza (\%) & $11.95 \mathrm{a}$ & $11.89 \mathrm{a}$ & $12.28 \mathrm{a}$ & 0.049 & 2.76 \\
Materia orgánica (\%) & $85.23 \mathrm{a}$ & $85.03 \mathrm{a}$ & $85.31 \mathrm{a}$ & 0.574 & 0.67
\end{tabular}

Medias con una letra común, no son significativamente diferentes (Tukey, p>0.05)

dentro de lo deseado.

Efecto edad de cosecha. La composición química influenciado por la edad de cosecha presentó diferencias $(\mathrm{p}<0.01)$, en proteína y materia orgánica destacándose la edad de 28 días, mientras, en materia seca se destaca la fenología 42 días (Cuadro 4), demostrándose que a menor edad el pasto mombaza 
Cuadro 4. Efecto de la edad de cosecha sobre la composición química del pasto mombaza (Panicum maximum Jacq).

\begin{tabular}{|c|c|c|c|c|c|}
\hline \multirow{2}{*}{ Variable } & \multicolumn{3}{|c|}{ Edades de cosecha (días) } & \multirow{2}{*}{$\mathbf{P}<$} & \multirow{2}{*}{ CV $(\%)$} \\
\hline & 28 & 35 & 42 & & \\
\hline Proteína (\%) & $9.23 \mathrm{a}$ & $8.31 \mathrm{~b}$ & $8.65 \mathrm{ab}$ & 0.004 & 6.16 \\
\hline MS (\%) & $29.33 \mathrm{c}$ & $29.78 \mathrm{~b}$ & $30.20 \mathrm{a}$ & 0.000 & 0.51 \\
\hline Ceniza (\%) & $11.93 \mathrm{a}$ & $12.15 \mathrm{a}$ & $12.05 \mathrm{a}$ & 0.371 & 2.76 \\
\hline Materia orgánica (\%) & $85.84 \mathrm{a}$ & $84.55 \mathrm{~b}$ & $85.18 \mathrm{ab}$ & 0.001 & 0.67 \\
\hline
\end{tabular}

Medias con una letra común, no son significativamente diferentes (Tukey, p>0.05)

es más nutritivo, atribuyéndose este efecto a lo manifestado por Ramírez et al. (2010) quienes indican que todo aquello está determinado por la radiación fotosintéticamente activa absorbida y por su eficacia de conversión a materia seca.

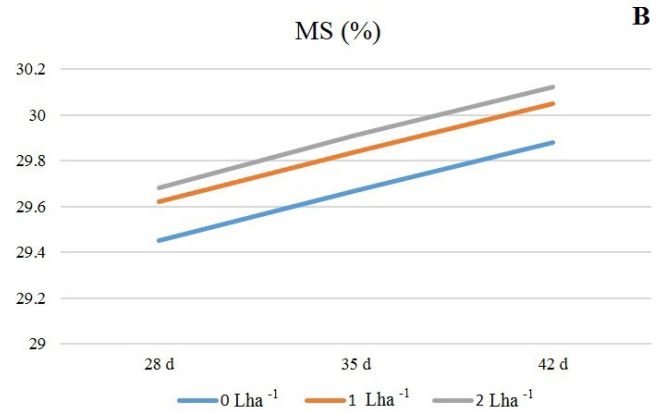

C
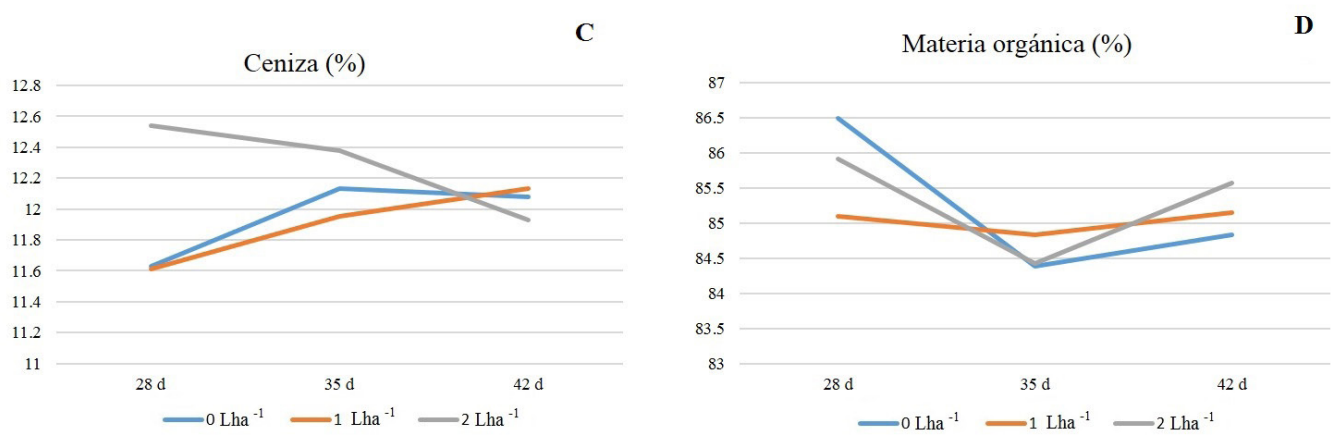

Figura 2. Efecto interacción en los contenidos de A) proteína, B) materia seca, C) ceniza y D) materia orgánica en la composición química del pasto mombaza

Efecto niveles de metalosato de zinc $x$ edades de cosecha. El efecto interacción entre los niveles de metalosato de zinc $\mathrm{x}$ edades de cosecha en la composición química, mostraron una tendencia similar en proteina, ceniza y materia orgánica cuando se aplicó 0 y $1 \mathrm{~L} \mathrm{ha}^{-1}$ a los 28 hasta los 35 días de cosecha, consecuentemente de ahi en adelante la variable respuesta no depende del factor de estudio. En cuanto a la MS, tuvo un incremento en los factores en estudio, mostrándose dependencia entre la variable respuesta con respecto al factor de estudio (Figura 2).

\section{Conclusiones}

Zl uso del metalosato de zinc influenció positivamente en Cel peso de hoja (5.4 g), peso de tallo (4.76 g), longitud de hoja $(55.39 \mathrm{~cm})$, biomasa $\left(3369.76 \mathrm{MS} \mathrm{kg} \mathrm{ha}^{-1}\right)$ y materia seca $(30.03 \%)$, incrementándose su contenido hasta cuando se agregó $2 \mathrm{~L} \mathrm{ha}^{-1}$ de metalosato de zinc a los 28 y 42 dias de cosecha. 


\section{Bibliografía}

Cerdas, R. (2011). Programa de fertilización de forrajes. Desarrollo de un módulo práctico para técnicos y estudiantes de ganadería de Guanacaste, Costa Rica. InterSedes: Revista de las Sedes Regionales, 12(12): 109-128.

Chaves, M., y Barrantes, JC. (1999). Efecto del zinc aplicado al suelo y foliar, sobre la producción agroindustrial de la variedad de caña de azúcar sp 71-5574 en un ultisol de Pérez Zeledón. Promedio de cuatro cosechas. Memoria San José, Asociación de técnicos azucareros de Costa Rica (ATACORI).

Di Rienzo, JA., Casanoves, F., Balzarini, MG., Gonzalez, L., Tablada, M., Robledo, CW. (2008). InfoStat, versión 2008, Grupo InfoStat, FCA, Universidad Nacional de Córdoba, Argentina.

García, C., Martínez, R., Tuero, R., Cruz, A., Romero, A., Estanquero, L., Noda, A. y Torres, V. (2008). Evaluación agronómica de Guinea Mombaza (Panicum máximum Jacq) en un suelo ferralítico rojo típico de la provincia La Habana. Revista Cubana de Ciencia Agrícola, 42(2): 205-209.

Garmendia, J. (1998). Suplementación estratégica en la reproducción de vacas de doble propósito. En: T. Clavero (Ed.). Estrategias de Alimentación para la Ganadería Tropical. Centro de Transferencia de Tecnología en Pastos y Forrajes. LUZ. Maracaibo. 43-52 pp.

González-Dominguez, M. (2016). Patologías dermatológicas de origen nutricional en los pequeños animales: una revisión. Revista CES Medicina Veterinaria y Zootecnia, 11(2): 82-102.

La O, O., González, H., Orozco, A., Castillo, Y., Ruiz, O., Estrada, A., Ríos, F., Gutiérrez, E., Bernal, H., Valenciaga, D., Castro, B. y Hernández, Y. (2012). Composición química, degradabilidad ruminal in situ y digestibilidad in vitro de ecotipos de Tithonia diversifolia de interés para la alimentación de rumiantes. Revista Cubana de Ciencia Agrícola, 46(1).

Müller, MS., Fancelli, AL., Dourado-Neto, D., García, A. \& Ovejero, RF. (2002). Produtividade do Panicum máximum cv. Mombaça irrigado, sobpaste jorotacionado. Scientia Agricola 59(3): 427-433.

Nowack, B. (2002). Environmental Chemistry of Aminopolycarboxylate Chelating Agents, Environ. Sci. Technol., 36, pp. 4009 - 4016.

Paulino, V. (2004). Potencialidade de pasta genstropicais para produção animal. Simpósio de Produção Animal a Pasto no Norte Pioneiro. R. Bras. Zootec. 33:56.

Ramírez, O., Hernandez, A., Carneiro, S., Perez, J., Jacaúna, S., Castro, R. y Enríquez, J. (2010). Características morfogenéticas y su influencia en el rendimiento del pasto mombaza, cosechado a diferentes intervalos de corte. Tropical and Subtropical Agroecosystems, 12: 303-311.

Vargas, J., Leonard, I., Uvidia, H., Ramírez, J., Torres, V., Andino, M. y Benítez, D. (2014). El crecimiento del pasto Panicum maximum vc Mombaza en la Amazonía Ecuatoriana. REDVET - Revista electrónica de Veterinaria - 15(9): 1-7.

Verdecia, D., Herrera, R., Ramírez, J., Leonard, I., Bodas, R., Andrés, S., Giráldez, F., Álvarez, Y. y López, S. (2012). Valoración nutritiva del Panicum maximum vc. Mombasa en las condiciones climáticas del Valle del Cauto, Cuba. Revista Cubana de Ciencia Agrícola, 46(1): 97-101

Verdecia, D., Ramírez, J., Leonard, I. y García, F. (2009). Potencialidades agroproductivas de dos cultivares de Panicum maximum (c.v Mombasa y Uganda) en la provincia Granma. REDVET. Revista electrónica de Veterinaria. 10(5) 1-9.

Villalobos, C., Gonzáles, E. y Ortega, J. (2000). Técnicas para estimar la degradación de proteína y materia orgánica en el rumen y su importancia en rumiantes en pastoreo. Técnica Pecuaria en México, 38(2): 119-134. 